\title{
Unveiling the full potential of flexible receptor docking using multiple crystallographic structures.
}

\author{
Xavier Barril and S. David Morley
}

Contents: Tables S.1 and S.2 contain the PDB codes of the structures used in this study, with its corresponding references, information about the nature of the ligand, the volume of the docking cavities and performance of the cavities in SC- and MC-docking. Figures S.1 and S.2 illustrate examples of ligands in the test sets.

Table S.1: List of CDK2 cavities used in this study. PDB codes, with its corresponding reference, the nature of the ligand, the volume of the docking site and the docking performance values are provided.

\begin{tabular}{|c|c|c|c|c|c|c|c|}
\hline \multirow[t]{2}{*}{$\begin{array}{l}\text { Cavity } \\
\text { ID }\end{array}$} & \multirow[t]{2}{*}{$\begin{array}{l}\text { PDB } \\
\text { code }\end{array}$} & \multirow[t]{2}{*}{ Reference } & \multirow[t]{2}{*}{$\begin{array}{l}\text { Ligand } \\
\text { Type }\end{array}$} & \multirow[t]{2}{*}{$\begin{array}{l}\text { Volume of } \\
\text { Docking } \\
\text { Cavity }\left(\AA^{3}\right)\end{array}$} & \multirow[t]{2}{*}{$\begin{array}{c}\text { SC-Docking } \\
\text { Performance } \\
\text { (\%) }\end{array}$} & \multicolumn{2}{|c|}{$\begin{array}{l}\text { Contribution to } \\
\text { MC-Docking } \\
\text { Performance } \\
\text { (6 cavities) }\end{array}$} \\
\hline & & & & & & $\begin{array}{c}\text { Average } \\
(\%)\end{array}$ & $\begin{array}{l}\text { Std. } \\
\text { Dev. }\end{array}$ \\
\hline 1 & $1 \mathrm{aq} 1$ & 1 & Organic & 1440.75 & 47.06 & 9.92 & 16.96 \\
\hline 2 & $1 \mathrm{~b} 38$ & 2 & ATP $+M g$ & 1149.62 & 29.41 & 15.01 & 13.91 \\
\hline 3 & $1 \mathrm{~b} 39$ & 2 & ATP+Mg & 1204.75 & 20.59 & 3.93 & 3.96 \\
\hline 4 & 1 buh & 3 & Apo & 1214.62 & 2.94 & 1.98 & 3.87 \\
\hline 5 & 1 ckp & 4 & Organic & 1248.38 & 55.88 & 14.43 & 23.85 \\
\hline 6 & $1 \mathrm{di} 8$ & 5 & Organic & 1362.75 & 50.00 & 4.56 & 15.91 \\
\hline 7 & $1 \mathrm{dm} 2$ & 6 & Organic & 1319.88 & 26.47 & -1.06 & 8.70 \\
\hline 8 & $1 \mathrm{e} 1 \mathrm{v}$ & 7 & Organic & 1021.25 & 23.53 & 8.57 & 14.59 \\
\hline 9 & $1 \mathrm{e} 1 \mathrm{x}$ & 7 & Organic & 1096.88 & 26.47 & 10.75 & 15.87 \\
\hline 10 & $1 \mathrm{e} 9 \mathrm{~h}$ & 8 & Organic & 1650.75 & 26.47 & 2.35 & 8.46 \\
\hline 11 & $1 \mathrm{fin}$ & 9 & ATP & 1787.50 & 44.12 & 2.64 & 10.91 \\
\hline 12 & $1 \mathrm{fq} 1$ & 10 & $\mathrm{ATP}+\mathrm{Mg}$ & 1460.62 & 2.94 & -5.89 & 7.62 \\
\hline 13 & $1 \mathrm{fvt}$ & 11 & Organic & 1409.00 & 50.00 & 4.94 & 14.16 \\
\hline 14 & $1 \mathrm{~g} 5 \mathrm{~s}$ & 12 & Organic & 1533.50 & 64.71 & 12.62 & 18.65 \\
\hline 15 & 1 gih & 13 & Organic & 1364.62 & 52.94 & 6.57 & 20.54 \\
\hline 16 & 1 1gy3 & 14 & $\mathrm{ATP}+\mathrm{Mg}$ & 1667.88 & 8.82 & -0.33 & 3.91 \\
\hline 17 & $1 \mathrm{gz} 8$ & 15 & Organic & 1196.50 & 26.47 & 5.77 & 9.00 \\
\hline 18 & $1 \mathrm{hOu}$ & ${ }^{*}$ & Organic & 1158.38 & 23.53 & 1.89 & 4.49 \\
\hline 19 & $1 \mathrm{~h} 0 \mathrm{v}$ & 15 & Organic & 1079.12 & 26.47 & 14.20 & 14.90 \\
\hline 20 & $1 \mathrm{hOw}$ & 15 & Organic & 1346.62 & 38.24 & 2.91 & 6.09 \\
\hline 21 & $1 \mathrm{~h} 1 \mathrm{p}$ & 16 & Organic & 1224.38 & 23.53 & -3.13 & 15.22 \\
\hline 22 & $1 \mathrm{~h} 1 \mathrm{q}$ & 16 & Organic & 1284.75 & 38.24 & 23.33 & 18.06 \\
\hline 23 & $1 \mathrm{~h} 1 \mathrm{r}$ & 16 & Organic & 1332.25 & 32.35 & 10.70 & 30.22 \\
\hline 24 & $1 \mathrm{~h} 1 \mathrm{~s}$ & 16 & Organic & 1377.12 & 58.82 & 13.15 & 24.97 \\
\hline 25 & $1 \mathrm{~h} 24$ & 17 & Apo & 1801.12 & 32.35 & 12.08 & 11.99 \\
\hline 26 & $1 \mathrm{~h} 25$ & 17 & Apo & 1568.75 & 26.47 & 2.02 & 10.74 \\
\hline 27 & $1 \mathrm{~h} 26$ & 17 & Apo & 1640.12 & 26.47 & -8.28 & 10.05 \\
\hline 28 & $1 \mathrm{~h} 27$ & 17 & Apo & 1640.88 & 17.65 & 0.70 & 3.92 \\
\hline 29 & $1 \mathrm{~h} 28$ & 17 & Apo & 1659.25 & 14.71 & -11.68 & 10.49 \\
\hline
\end{tabular}


Supporting Information

\begin{tabular}{|c|c|c|c|c|c|c|c|}
\hline 30 & 1hck & 18 & $\mathrm{ATP}+\mathrm{Mg}$ & 1199.00 & 29.41 & 8.23 & 8.54 \\
\hline 31 & $1 \mathrm{hcl}$ & 19 & Apo & 1318.88 & 2.94 & -0.76 & 1.83 \\
\hline 32 & 1jst & 20 & ATP+Mn & 1600.88 & 29.41 & 0.26 & 1.81 \\
\hline 33 & $1 \mathrm{jsv}$ & 21 & Organic & 1330.62 & 29.41 & 1.46 & 3.49 \\
\hline 34 & $1 \mathrm{jvp}$ & 22 & Organic & 1365.25 & 35.29 & -4.27 & 16.06 \\
\hline 35 & $1 \mathrm{ke5}$ & 23 & Organic & 1274.00 & 52.94 & 9.67 & 25.40 \\
\hline 36 & $1 \mathrm{ke} 6$ & 23 & Organic & 1408.62 & 67.65 & 9.99 & 18.94 \\
\hline 37 & $1 \mathrm{ke} 7$ & 23 & Organic & 1310.12 & 55.88 & 7.59 & 16.83 \\
\hline 38 & $1 \mathrm{ke} 8$ & 23 & Organic & 1288.50 & 50.00 & 9.28 & 19.71 \\
\hline 39 & $1 \mathrm{ke} 9$ & 23 & Organic & 1325.50 & 41.18 & 5.51 & 18.20 \\
\hline 40 & $\log u$ & 24 & Organic & 1289.88 & 64.71 & 24.37 & 24.96 \\
\hline 41 & 10it & 25 & Organic & 1314.50 & 50.00 & 7.32 & 13.18 \\
\hline 42 & $1 p 5 e$ & 26 & Organic & 1598.00 & 32.35 & 0.95 & 5.17 \\
\hline 43 & $1 \mathrm{pkd}$ & 27 & Organic & 1740.25 & 38.24 & 7.17 & 12.53 \\
\hline 44 & $1 p w 2$ & 28 & Apo & 1143.25 & 2.94 & -0.49 & 1.61 \\
\hline 45 & $1 p x i$ & 28 & Organic & 1127.50 & 2.94 & -1.98 & 3.96 \\
\hline 46 & $1 p x j$ & 28 & Organic & 1226.62 & 17.65 & -0.40 & 1.47 \\
\hline 47 & $1 \mathrm{pxk}$ & 28 & Organic & 1250.75 & 41.18 & 6.15 & 13.85 \\
\hline 48 & $1 p x \mid$ & 28 & Organic & 1210.25 & 52.94 & 5.59 & 17.42 \\
\hline 49 & $1 \mathrm{qmz}$ & 29 & $\mathrm{ATP}+\mathrm{Mg}$ & 1529.62 & 20.59 & 2.53 & 6.55 \\
\hline \multicolumn{4}{|c|}{ Subset } & $\begin{array}{c}\text { Average } \pm \\
\text { St.Dev. }\end{array}$ & $\begin{array}{c}\text { Average } \pm \\
\text { St.Dev. }\end{array}$ & $\begin{array}{c}\text { Average } \pm \\
\text { St.Dev. }\end{array}$ & \\
\hline \multicolumn{4}{|c|}{ Apo CDK2 } & $1498 \pm 240$ & $15.81 \pm 11.97$ & $-0.55 \pm 7.15$ & \\
\hline \multicolumn{4}{|c|}{ CDK2-ATP } & $1450 \pm 240$ & $23.16 \pm 13.01$ & $3.30 \pm 6.21$ & \\
\hline \multicolumn{4}{|c|}{ CDK2-Organic } & $1318 \pm 155$ & $40.11 \pm 15.47$ & $7.00 \pm 6.58$ & \\
\hline \multicolumn{4}{|c|}{ All } & $1369 \pm 196$ & $33.37 \pm 17.47$ & $5.16 \pm 7.09$ & \\
\hline
\end{tabular}

* This entry was deleted from the PDB 
Table S.2: List of HSP90 cavities used in this study. PDB codes, with its corresponding reference (when available), the nature of the ligand, the fold adopted by the flexible loop, the volume of the docking site and the docking performances are provided.

\begin{tabular}{|c|c|c|c|c|c|c|c|c|}
\hline \multirow[t]{2}{*}{$\begin{array}{l}\text { Cavity } \\
\text { ID }\end{array}$} & \multirow[t]{2}{*}{$\begin{array}{l}\text { PDB } \\
\text { code }\end{array}$} & \multirow[t]{2}{*}{ Ref. } & \multirow[t]{2}{*}{$\begin{array}{l}\text { Ligand } \\
\text { Type }\end{array}$} & \multirow[t]{2}{*}{$\begin{array}{l}\text { Loop } \\
\text { Confor- } \\
\text { mation }\end{array}$} & \multirow{2}{*}{$\begin{array}{c}\text { Cavity } \\
\text { Volume } \\
\left(\AA^{3}\right)\end{array}$} & \multirow[t]{2}{*}{$\begin{array}{c}\text { SC-Docking } \\
\text { Performance } \\
(\%)\end{array}$} & \multicolumn{2}{|c|}{$\begin{array}{l}\text { Contribution to } \\
\text { MC-Docking } \\
\text { Performance } \\
\text { (6 cavities) }\end{array}$} \\
\hline & & & & & & & $\begin{array}{c}\text { Average } \\
(\%)\end{array}$ & $\begin{array}{c}\text { Average } \\
(\%)\end{array}$ \\
\hline 1 & 1 byq & 30 & $\mathrm{ADP}+\mathrm{Mg}$ & Open & 3254.62 & 12.28 & 4.04 & 5.42 \\
\hline 2 & $10 s f$ & 31 & Organic & Open & 3206.50 & 12.28 & 3.21 & 4.95 \\
\hline 3 & 1yes & 32 & Apo & Open & 3315.12 & 7.02 & -0.48 & 2.93 \\
\hline 4 & 1yet & 32 & Organic & Open & 3300.00 & 15.79 & 8.12 & 9.42 \\
\hline 5 & n.a. & n.a. & Organic & Open & 3218.12 & 35.09 & 3.79 & 7.77 \\
\hline 6 & n.a. & n.a. & Organic & Open & 3212.25 & 40.35 & 11.41 & 22.21 \\
\hline 7 & n.a. & n.a. & Organic & Open & 3223.50 & 42.11 & 16.59 & 22.80 \\
\hline 8 & n.a. & n.a. & ADPnP & Open & 3189.38 & 10.53 & 3.10 & 3.42 \\
\hline 9 & n.a. & n.a. & Organic & Helical & 3066.25 & 38.60 & 3.61 & 9.02 \\
\hline 10 & 1uy6 & 33 & Organic & Helical & 3163.38 & 12.28 & 6.44 & 11.17 \\
\hline 11 & n.a. & n.a. & Organic & Helical & 3202.50 & 22.81 & 7.51 & 10.44 \\
\hline 12 & 1uy7 & 33 & Organic & Helical & 3210.00 & 15.79 & 6.26 & 9.93 \\
\hline 13 & n.a. & n.a. & Organic & Helical & 3204.50 & 3.51 & -3.06 & 4.40 \\
\hline 14 & n.a. & n.a. & Organic & Helical & 2823.00 & 15.79 & 2.07 & 7.13 \\
\hline 15 & n.a. & n.a. & Organic & Helical & 3181.12 & 10.53 & -1.25 & 4.76 \\
\hline 16 & n.a. & n.a. & Organic & Helical & 3162.62 & 8.77 & -5.67 & 6.66 \\
\hline 17 & n.a. & n.a. & Organic & Helical & 3145.62 & 17.54 & -0.50 & 6.36 \\
\hline 18 & n.a. & n.a. & Organic & Helical & 3223.12 & 19.30 & 9.00 & 10.87 \\
\hline 19 & n.a. & n.a. & Organic & Helical & 3239.38 & 14.04 & 2.26 & 11.72 \\
\hline 20 & n.a. & n.a. & Organic & Helical & 3276.50 & 33.33 & 9.56 & 8.74 \\
\hline 21 & n.a. & n.a. & Organic & Helical & 3065.75 & 26.32 & 1.00 & 9.30 \\
\hline 22 & n.a. & n.a. & Organic & Helical & 3192.25 & 29.83 & 6.80 & 11.14 \\
\hline 23 & n.a. & n.a. & Organic & Helical & 3257.62 & 17.54 & 6.34 & 7.17 \\
\hline 24 & 1uy8 & 33 & Organic & Helical & 3243.88 & 15.79 & 7.62 & 9.96 \\
\hline 25 & n.a. & n.a. & Organic & Helical & 3213.12 & 14.04 & 1.89 & 10.59 \\
\hline 26 & 1uye & 33 & Organic & Helical & 3269.62 & 14.04 & 7.05 & 9.69 \\
\hline 27 & 1uyd & 33 & Organic & Helical & 3275.00 & 15.79 & 7.84 & 10.26 \\
\hline 28 & n.a. & n.a. & Organic & Helical & 3113.38 & 3.51 & -0.20 & 0.96 \\
\hline 29 & n.a. & n.a. & Organic & Helical & 3047.12 & 29.83 & 3.53 & 11.78 \\
\hline 30 & 1uy9 & 33 & Organic & Helical & 3205.38 & 15.79 & 7.89 & 9.93 \\
\hline 31 & 1uyc & 33 & Organic & Helical & 3206.12 & 14.04 & 7.23 & 9.71 \\
\hline 32 & n.a. & n.a. & Organic & Helical & 3120.75 & 17.54 & 7.88 & 10.13 \\
\hline 33 & n.a. & n.a. & Organic & Helical & 3160.12 & 12.28 & 3.76 & 6.88 \\
\hline 34 & 1uyf & 33 & Organic & Helical & 3265.25 & 14.04 & 5.91 & 10.08 \\
\hline 35 & n.a. & n.a. & Organic & Helical & 3034.88 & 28.07 & 5.69 & 11.50 \\
\hline 36 & n.a. & n.a. & Organic & Helical & 3208.75 & 17.54 & 11.26 & 11.05 \\
\hline 37 & n.a. & n.a. & Organic & Helical & 3108.50 & 7.02 & 0.34 & 3.24 \\
\hline 38 & n.a. & n.a. & Organic & Helical & 3387.75 & 10.53 & -0.03 & 2.16 \\
\hline 39 & n.a. & n.a. & Organic & Helical & 3151.25 & 7.02 & -1.70 & 2.93 \\
\hline 40 & 1uyg & 33 & Organic & Helical & 3220.62 & 14.04 & 4.98 & 9.00 \\
\hline 41 & 1uyh & 33 & Organic & Helical & 3207.88 & 14.04 & 8.27 & 10.12 \\
\hline
\end{tabular}


Supporting Information

\begin{tabular}{|c|c|c|c|c|c|c|c|c|}
\hline 42 & n.a. & n.a. & Organic & Helical & 3257.50 & 14.04 & 6.05 & 8.53 \\
\hline 43 & 1uyi & 33 & Organic & Helical & 3174.12 & 14.04 & 7.36 & 9.13 \\
\hline 44 & n.a. & n.a. & Organic & Helical & 3269.88 & 19.30 & 1.13 & 8.24 \\
\hline 45 & 1uyk & 33 & Organic & Helical & 3238.00 & 10.53 & -0.91 & 4.06 \\
\hline 46 & n.a. & n.a. & Organic & Helical & 3062.75 & 3.51 & 0.00 & 0.00 \\
\hline 47 & n.a. & n.a. & Organic & Helical & 3095.12 & 21.05 & 4.06 & 6.36 \\
\hline 48 & n.a. & n.a. & Organic & Helical & 3188.62 & 15.79 & 1.06 & 7.70 \\
\hline 49 & n.a. & n.a. & Organic & Helical & 3124.00 & 14.04 & 4.51 & 5.32 \\
\hline 50 & n.a. & n.a. & Organic & Helical & 3207.88 & 15.79 & 4.44 & 9.98 \\
\hline 51 & n.a. & n.a. & Organic & Helical & 3210.88 & 22.81 & 8.08 & 6.53 \\
\hline 52 & n.a. & n.a. & Organic & Helical & 3213.88 & 28.07 & 11.95 & 9.52 \\
\hline 53 & n.a. & n.a. & Organic & Helical & 3116.75 & 28.07 & 1.75 & 5.88 \\
\hline 54 & n.a. & n.a. & Organic & Helical & 3091.88 & 12.28 & 0.10 & 4.36 \\
\hline 55 & n.a. & n.a. & Organic & Helical & 3200.00 & 14.04 & 3.71 & 4.62 \\
\hline 56 & n.a. & n.a. & Organic & Helical & 3093.25 & 10.53 & -2.34 & 4.79 \\
\hline 57 & n.a. & n.a. & Organic & Helical & 3175.62 & 10.53 & -2.53 & 5.41 \\
\hline 58 & n.a. & n.a. & Organic & Helical & 3155.75 & 15.79 & 4.04 & 9.32 \\
\hline 59 & n.a. & n.a. & Organic & Helical & 3174.62 & 3.51 & -6.79 & 6.08 \\
\hline 60 & n.a. & n.a. & Organic & Helical & 3101.50 & 10.53 & 2.57 & 8.74 \\
\hline 61 & n.a. & n.a. & Organic & Helical & 3179.00 & 15.79 & 2.92 & 7.18 \\
\hline 62 & n.a. & n.a. & Organic & Helical & 3231.88 & 10.53 & -3.27 & 5.51 \\
\hline 63 & n.a. & n.a. & Organic & Helical & 3206.50 & 5.26 & -7.78 & 7.01 \\
\hline 64 & n.a. & n.a. & Organic & Helical & 3174.12 & 21.05 & 8.21 & 7.18 \\
\hline 65 & n.a. & n.a. & Organic & Helical & 3062.12 & 0.00 & -2.17 & 3.51 \\
\hline 66 & n.a. & n.a. & Organic & Helical & 3128.62 & 17.54 & 5.89 & 9.32 \\
\hline 67 & n.a. & n.a. & Organic & Helical & 3269.00 & 10.53 & -0.76 & 2.76 \\
\hline 68 & 1 yer & 32 & Apo & Closed & 2845.25 & 31.58 & 3.11 & 8.81 \\
\hline 69 & n.a. & n.a. & Organic & Closed & 2862.25 & 38.60 & 12.36 & 19.59 \\
\hline 70 & n.a. & n.a. & Organic & Closed & 2820.00 & 31.58 & 5.84 & 14.21 \\
\hline 71 & n.a. & n.a. & Organic & Closed & 2849.12 & 0.00 & -0.25 & 1.14 \\
\hline 72 & n.a. & n.a. & Organic & Closed & 3133.12 & 36.84 & 5.31 & 10.08 \\
\hline 73 & n.a. & n.a. & Organic & Closed & 2810.38 & 7.02 & -2.40 & 4.76 \\
\hline 74 & n.a. & n.a. & Organic & Closed & 2765.88 & 12.28 & 5.65 & 6.52 \\
\hline 75 & n.a. & n.a. & Organic & Closed & 2913.50 & 10.53 & 1.14 & 5.47 \\
\hline 76 & n.a. & n.a. & Organic & Closed & 2868.62 & 10.53 & 2.97 & 4.96 \\
\hline 77 & n.a. & n.a. & Organic & Closed & 2769.62 & 10.53 & -0.53 & 5.33 \\
\hline 78 & n.a. & n.a. & Organic & Closed & 2872.25 & 35.09 & 2.64 & 12.34 \\
\hline 79 & n.a. & n.a. & Organic & Closed & 2818.12 & 14.04 & 0.78 & 6.93 \\
\hline 80 & n.a. & n.a. & Organic & Closed & 2865.12 & 40.35 & 9.91 & 22.07 \\
\hline 81 & n.a. & n.a. & Organic & Closed & 2890.88 & 31.58 & 2.50 & 10.89 \\
\hline 82 & n.a. & n.a. & Organic & Closed & 2853.62 & 43.86 & 12.03 & 25.20 \\
\hline 83 & n.a. & n.a. & Organic & Closed & 2857.25 & 40.35 & 7.65 & 16.26 \\
\hline 84 & n.a. & n.a. & Organic & Closed & 2856.00 & 22.81 & 0.69 & 4.85 \\
\hline 85 & n.a. & n.a. & Organic & Closed & 2886.12 & 45.61 & 15.21 & 27.93 \\
\hline 86 & n.a. & n.a. & Organic & Closed & 2943.12 & 45.61 & 23.51 & 30.74 \\
\hline 87 & n.a. & n.a. & Organic & Closed & 2833.50 & 47.37 & 21.52 & 27.53 \\
\hline 88 & n.a. & n.a. & Organic & Closed & 2906.75 & 29.83 & 1.59 & 8.16 \\
\hline 89 & n.a. & n.a. & Organic & Closed & 2969.50 & 36.84 & 11.11 & 22.81 \\
\hline 90 & n.a. & n.a. & Organic & Closed & 2834.62 & 40.35 & 4.03 & 14.13 \\
\hline 91 & n.a. & n.a. & Organic & Closed & 2820.00 & 38.60 & 4.17 & 18.11 \\
\hline
\end{tabular}


Supporting Information

\begin{tabular}{|c|c|c|c|c|c|c|c|c|}
\hline 92 & n.a. & n.a. & Organic & Closed & 2801.50 & 28.07 & 3.23 & 10.17 \\
\hline 93 & n.a. & n.a. & Organic & Closed & 2820.12 & 43.86 & 10.20 & 23.85 \\
\hline 94 & n.a. & n.a. & Organic & Closed & 2846.25 & 42.11 & 5.87 & 16.76 \\
\hline 95 & n.a. & n.a. & Organic & Closed & 2802.00 & 8.77 & -2.04 & 4.12 \\
\hline 96 & n.a. & n.a. & Organic & Closed & 2868.12 & 43.86 & 11.98 & 21.30 \\
\hline 97 & n.a. & n.a. & Organic & Closed & 2939.12 & 45.61 & 14.39 & 24.57 \\
\hline 98 & n.a. & n.a. & Organic & Closed & 2797.50 & 36.84 & 15.00 & 25.19 \\
\hline 99 & n.a. & n.a. & Organic & Closed & 2767.62 & 31.58 & 3.67 & 13.14 \\
\hline 100 & n.a. & n.a. & Organic & Closed & 2828.25 & 31.58 & 10.76 & 24.73 \\
\hline 101 & n.a. & n.a. & Organic & Closed & 2892.00 & 35.09 & 6.14 & 15.35 \\
\hline 102 & n.a. & n.a. & Organic & Closed & 2902.62 & 33.33 & -0.52 & 5.37 \\
\hline 103 & n.a. & n.a. & Organic & Closed & 2832.25 & 35.09 & 3.13 & 14.04 \\
\hline 104 & n.a. & n.a. & Organic & Closed & 2832.12 & 36.84 & 5.15 & 13.80 \\
\hline 105 & n.a. & n.a. & Organic & Closed & 2837.12 & 40.35 & 6.91 & $\overline{17.40}$ \\
\hline 106 & n.a. & n.a. & Organic & Closed & 2805.25 & 40.35 & 7.93 & 22.34 \\
\hline 107 & n.a. & n.a. & Organic & Closed & 2876.12 & 45.61 & 8.29 & 20.94 \\
\hline 108 & n.a. & n.a. & Organic & Closed & 2806.38 & 33.33 & 7.54 & 20.33 \\
\hline 109 & n.a. & n.a. & Organic & Closed & 2812.50 & 12.28 & 5.54 & 7.85 \\
\hline 110 & n.a. & n.a. & Organic & Closed & 2817.25 & 8.77 & -2.91 & 3.09 \\
\hline 111 & n.a. & n.a. & Organic & Closed & 2760.62 & 7.02 & -1.27 & 2.71 \\
\hline 112 & n.a. & n.a. & Organic & Closed & 2827.38 & 24.56 & 0.73 & 11.94 \\
\hline 113 & n.a. & n.a. & Organic & Closed & 2870.25 & 33.33 & 9.86 & 18.66 \\
\hline 114 & n.a. & n.a. & Organic & Closed & 2925.88 & 40.35 & 3.84 & 17.18 \\
\hline 115 & n.a. & n.a. & Organic & Closed & 2932.75 & 43.86 & 8.03 & 19.18 \\
\hline 116 & n.a. & n.a. & Organic & Closed & 2918.88 & 42.11 & 11.87 & 24.86 \\
\hline 117 & n.a. & n.a. & Organic & Closed & 2922.50 & 45.61 & 22.88 & 29.78 \\
\hline 118 & n.a. & n.a. & Organic & Closed & 2877.50 & 31.58 & 6.04 & 19.16 \\
\hline 119 & n.a. & n.a. & Organic & Closed & 2905.00 & 22.81 & 2.43 & 4.76 \\
\hline 120 & n.a. & n.a. & Organic & Closed & 2862.88 & 40.35 & 19.77 & 33.27 \\
\hline 121 & n.a. & n.a. & Organic & Closed & 2903.00 & 31.58 & 6.22 & 15.73 \\
\hline 122 & n.a. & n.a. & Organic & Closed & 2860.75 & 40.35 & 14.22 & 25.83 \\
\hline 123 & n.a. & n.a. & Organic & Closed & 2871.12 & 36.84 & 6.64 & 13.94 \\
\hline 124 & n.a. & n.a. & Organic & Closed & 2827.12 & 12.28 & 1.03 & 5.93 \\
\hline 125 & n.a. & n.a. & Organic & Closed & 2892.62 & 40.35 & 12.59 & 24.73 \\
\hline 126 & n.a. & n.a. & Organic & Closed & 2899.38 & 40.35 & 11.44 & 22.32 \\
\hline 127 & n.a. & n.a. & Organic & Closed & 2878.12 & 40.35 & 10.28 & 23.67 \\
\hline 128 & n.a. & n.a. & Organic & Closed & 2881.62 & 42.11 & 12.95 & 24.87 \\
\hline 129 & n.a. & n.a. & Organic & Closed & 2935.88 & 38.60 & 4.96 & 16.86 \\
\hline 130 & n.a. & n.a. & Organic & Closed & 2842.12 & 35.09 & 5.09 & 12.76 \\
\hline 131 & n.a. & n.a. & Organic & Closed & 2881.38 & 42.11 & 12.71 & 23.57 \\
\hline 132 & n.a. & n.a. & Organic & Closed & 2895.25 & 38.60 & 9.61 & 23.40 \\
\hline 133 & n.a. & n.a. & Organic & Closed & 2893.75 & 47.37 & 20.20 & 32.39 \\
\hline 134 & n.a. & n.a. & Organic & Closed & 2947.12 & 49.12 & 19.12 & 31.17 \\
\hline 135 & n.a. & n.a. & Organic & Closed & 2866.25 & 33.33 & 2.56 & 10.04 \\
\hline 136 & n.a. & n.a. & Organic & Closed & 2894.25 & 42.11 & 10.69 & 25.23 \\
\hline 137 & n.a. & n.a. & Organic & Closed & 2966.75 & 42.11 & 7.22 & 18.42 \\
\hline 138 & n.a. & n.a. & Organic & Closed & 2987.50 & 45.61 & 13.34 & 24.95 \\
\hline 139 & n.a. & n.a. & Organic & Closed & 2999.00 & 43.86 & 13.32 & 23.48 \\
\hline 140 & n.a. & n.a. & Organic & Closed & 3013.50 & 36.84 & 11.80 & 23.05 \\
\hline 141 & n.a. & n.a. & Organic & Closed & 2881.25 & 49.12 & 24.09 & 34.38 \\
\hline
\end{tabular}


Supporting Information

\begin{tabular}{|c|c|c|c|c|c|c|c|c|}
\hline 142 & n.a. & n.a. & Organic & Closed & 2738.12 & 12.28 & 5.43 & 5.12 \\
\hline 143 & n.a. & n.a. & Organic & Closed & 2794.75 & 10.53 & 4.10 & 6.59 \\
\hline 144 & n.a. & n.a. & Organic & Closed & 2809.50 & 14.04 & 6.36 & 7.19 \\
\hline 145 & n.a. & n.a. & Organic & Closed & 2812.00 & 14.04 & 0.96 & 6.66 \\
\hline 146 & n.a. & n.a. & Organic & Closed & 2884.12 & 26.32 & 0.00 & 7.02 \\
\hline 147 & n.a. & n.a. & Apo & Closed & 2909.38 & 28.07 & 0.01 & 11.07 \\
\hline 148 & 1uyl & 33 & Apo & Closed & 2969.12 & 35.09 & 3.56 & 11.88 \\
\hline 149 & n.a. & n.a. & Organic & Closed & 2805.38 & 10.53 & -6.82 & 6.20 \\
\hline \multicolumn{5}{|c|}{ Subset } & $\begin{array}{l}\text { Average } \\
\pm \text { St.Dev. }\end{array}$ & $\begin{array}{c}\text { Average } \pm \\
\text { St.Dev. }\end{array}$ & $\begin{array}{l}\text { Average } \\
\pm \text { St.Dev. }\end{array}$ & \\
\hline \multicolumn{5}{|c|}{ HSP90-Organic } & $3007 \pm 171$ & $25.26 \pm 13.75$ & $5.78 \pm 6.10$ & \\
\hline \multicolumn{5}{|c|}{ Apo or ATP bound HSP90 } & $3080 \pm 198$ & $20.76 \pm 12.18$ & $2.22 \pm 1.94$ & \\
\hline \multicolumn{5}{|c|}{ Open Loop } & $3240 \pm 46$ & $21.93 \pm 14.62$ & $6.22 \pm 5.51$ & \\
\hline \multicolumn{5}{|c|}{ Helical Loop } & $3172 \pm 84$ & $15.52 \pm 7.69$ & $3.23 \pm 4.46$ & \\
\hline \multicolumn{5}{|c|}{ Closed Loop } & $2869 \pm 64$ & $32.26 \pm 12.71$ & $7.30 \pm 6.51$ & \\
\hline \multicolumn{5}{|c|}{ All } & $3010 \pm 172$ & $25.08 \pm 13.69$ & $5.63 \pm 6.02$ & \\
\hline
\end{tabular}


Figure S.1: Example of molecules binding to the ATP binding site of CDK2 used in this study. The molecules in the first row were used in the binding mode prediction experiments and the corresponding PDB code is provided. The molecules in the second and third row are part of the Deltagen set (J.Med.Chem. 2003, 46, 4360-4364) and were used in binding mode affinity prediction and VS experiments respectively. The activity is also provided.<smiles>CN[C@H]1CC2O[C@](C)([C@@H]1OC)n1c3ccccc3c3c4c(c5c6ccccc6n2c5c31)C(=O)NC4</smiles>

$1 \mathrm{AQ1}$<smiles>CCC(CO)Nc1nc(NCc2ccccc2)c2ncn(C(C)C)c2n1</smiles>

$\mathrm{IC}_{50}=0.087 \mathrm{uM}$<smiles>Cc1cnc(Nc2ccc3ncsc3c2)nc1NCC1CC1</smiles>

$\% \mathrm{I}=62.68$<smiles>Nc1nc(OCC2CCCCC2)c2nc[nH]c2n1</smiles>

1E1V<smiles>CCOC(=O)Nc1nc2cc(/C=C\c3ccc4c(c3)OCO4)ccc2[nH]1</smiles>

$\mathrm{IC}_{50}=1.83 \mathrm{uM}$<smiles>Oc1cc(O)cc(/C=C/c2ccc(O)c(O)c2)c1</smiles>

$\% \mathrm{l}=80.28$<smiles>NC1=NC(=O)/C(=C2\CCNC(=O)c3[nH]c(Br)cc32)N1</smiles>

1DM1<smiles>Oc1cccc(Nc2nc(-c3ccc4c(c3)OCO4)c(-c3ccc4c(c3)OCO4)s2)c1</smiles>

$\mathrm{IC}_{50}=96.0 \mathrm{uM}$<smiles>O=C(Nc1cc(Br)cc(NC(=O)N(c2ccccc2)c2ccccc2)c1)N(c1ccccc1)c1ccccc1</smiles>

$\% \mathrm{l}=51.9$ 
Figure S.2: Example of molecules binding to the ATP binding site of Hsp90 used in this study. All molecules were used in the binding mode prediction experiments. Molecules in the second and third row were used in binding affinity prediction. Molecules in the third row were used in VS experiments. When available, the $\mathrm{IC}_{50}$ values determined with a fluorometric assay ( $\mathrm{R}$. Howes et al.; unpublished results) are provided.<smiles>Nc1ncnc2c1ncn2[C@@H]1O[C@H](COP(=O)([O-])OP(=O)([O-])[O-])[C@@H](O)C1O</smiles><smiles>NC(=O)c1ccccc1N</smiles>

$\mathrm{IC}_{50}>4000 \mathrm{uM}$<smiles>COC(=O)c1cc(O)cc(O)c1</smiles>

$I_{50}=404 \mathrm{uM}$<smiles>Oc1cc(O)cc(CNc2cccc(OCc3ccccc3)c2)c1</smiles>

$I_{50}=294 u M$<smiles>C/C=C\C=C(\C)C(=O)NC1=CC(=O)C(OC)=C(C[C@@H](C)CC(OC)[C@@H](O)[C@@H](C)/C=C(\C)C(OC)C(C)OC)C1=O</smiles><smiles>Nc1ncnc2[nH]cnc12</smiles>

$\mathrm{IC}_{50}>4000 \mathrm{uM}$
Geldanamycin;<smiles>CCc1cc(-c2n[nH]cc2-c2ccccc2)c(O)cc1O</smiles>

$\mathrm{IC}_{50}=0.634 \mathrm{uM}$<smiles>C#CCCCn1c(Cc2cc(OC)ccc2OC)nc2c(N)nc(F)nc21</smiles>

$\mathrm{IC}_{50}=0.183 \mathrm{uM}$ $\mathrm{IC}_{50}=1.35 \mathrm{uM}$ 


\section{Supporting Information}

\section{References}

1. Lawrie, A. M.; Noble, M. E.; Tunnah, P.; Brown, N. R.; Johnson, L. N.; and Endicott, J. A. Protein kinase inhibition by staurosporine revealed in details of the molecular interaction with CDK2. Nat. Struct. Biol. 1997, 4, 796-801.

2. Brown, N. R.; Noble, M. E.; Lawrie, A. M.; Morris, M. C.; Tunnah, P.; Divita, G.; Johnson, L. N.; and Endicott, J. A. Effects of phosphorylation of threonine 160 on cyclindependent kinase 2 structure and activity. J. Biol. Chem. 1999, 274, 8746-8756.

3. Bourne, Y.; Watson, M. H.; Hickey, M. J.; Holmes, W.; Rocque, W.; Reed, S. I.; and Tainer, J. A. Crystal structure and mutational analysis of the human CDK2 kinase complex with cell cycle-regulatory protein CksHs1. Cell 1996, 84, 863-874.

4. Gray, N. S.; Wodicka, L.; Thunnissen, A. M.; Norman, T. C.; Kwon, S.; Espinoza, F. H.; Morgan, D. O.; Barnes, G.; LeClerc, S.; Meijer, L.; Kim, S. H.; Lockhart, D. J.; and Schultz, P. G. Exploiting chemical libraries, structure, and genomics in the search for kinase inhibitors. Science 1998, 281, 533-538.

5. Shewchuk, L.; Hassell, A.; Wisely, B.; Rocque, W.; Holmes, W.; Veal, J.; and Kuyper, L. F. Binding mode of the 4-anilinoquinazoline class of protein kinase inhibitor: X-ray crystallographic studies of 4-anilinoquinazolines bound to cyclin-dependent kinase 2 and p38 kinase. J. Med. Chem. 2000, 43, 133-138.

6. Meijer, L.; Thunnissen, A. M.; White, A. W.; Garnier, M.; Nikolic, M.; Tsai, L. H.; Walter, J.; Cleverley, K. E.; Salinas, P. C.; Wu, Y. Z.; Biernat, J.; Mandelkow, E. M.; Kim, S. H.; and Pettit, G. R. Inhibition of cyclin-dependent kinases, GSK-3beta and CK1 by hymenialdisine, a marine sponge constituent. Chem. Biol. 2000, 7, 51-63. 
7. Arris, C. E.; Boyle, F. T.; Calvert, A. H.; Curtin, N. J.; Endicott, J. A.; Garman, E. F.; Gibson, A. E.; Golding, B. T.; Grant, S.; Griffin, R. J.; Jewsbury, P.; Johnson, L. N.; Lawrie, A. M.; Newell, D. R.; Noble, M. E.; Sausville, E. A.; Schultz, R.; and Yu, W. Identification of novel purine and pyrimidine cyclin-dependent kinase inhibitors with distinct molecular interactions and tumor cell growth inhibition profiles. J. Med. Chem. 2000, 43, 2797-2804.

8. Davies, T. G.; Tunnah, P.; Meijer, L.; Marko, D.; Eisenbrand, G.; Endicott, J. A.; and Noble, M. E. Inhibitor binding to active and inactive CDK2: the crystal structure of CDK2-cyclin A/indirubin-5-sulphonate. Structure. (Camb. ) 2001, 9, 389-397.

9. Jeffrey, P. D.; Russo, A. A.; Polyak, K.; Gibbs, E.; Hurwitz, J.; Massague, J.; and Pavletich, N. P. Mechanism of CDK activation revealed by the structure of a cyclinACDK2 complex. Nature 1995, 376, 313-320.

10. Song, H.; Hanlon, N.; Brown, N. R.; Noble, M. E.; Johnson, L. N.; and Barford, D. Phosphoprotein-protein interactions revealed by the crystal structure of kinase-associated phosphatase in complex with phosphoCDK2. Mol. Cell 2001, 7, 615-626.

11. Davis, S. T.; Benson, B. G.; Bramson, H. N.; Chapman, D. E.; Dickerson, S. H.; Dold, K. M.; Eberwein, D. J.; Edelstein, M.; Frye, S. V.; Gampe Jr, R. T.; Griffin, R. J.; Harris, P. A.; Hassell, A. M.; Holmes, W. D.; Hunter, R. N.; Knick, V. B.; Lackey, K.; Lovejoy, B.; Luzzio, M. J.; Murray, D.; Parker, P.; Rocque, W. J.; Shewchuk, L.; Veal, J. M.; Walker, D. H.; and Kuyper, L. F. Prevention of chemotherapy-induced alopecia in rats by CDK inhibitors. Science 2001, 291, 134-137.

12. Dreyer, M. K.; Borcherding, D. R.; Dumont, J. A.; Peet, N. P.; Tsay, J. T.; Wright, P. S.; Bitonti, A. J.; Shen, J.; and Kim, S. H. Crystal structure of human cyclin-dependent 
kinase 2 in complex with the adenine-derived inhibitor H717. J. Med. Chem. 2001, 44, 524-530.

13. Ikuta, M.; Kamata, K.; Fukasawa, K.; Honma, T.; Machida, T.; Hirai, H.; SuzukiTakahashi, I.; Hayama, T.; and Nishimura, S. Crystallographic approach to identification of cyclin-dependent kinase 4 (CDK4)-specific inhibitors by using CDK4 mimic CDK2 protein. J. Biol. Chem. 2001, 276, 27548-27554.

14. Cook, A.; Lowe, E. D.; Chrysina, E. D.; Skamnaki, V. T.; Oikonomakos, N. G.; and Johnson, L. N. Structural studies on phospho-CDK2/cyclin A bound to nitrate, a transition state analogue: implications for the protein kinase mechanism. Biochemistry 2002, 41, 7301-7311.

15. Gibson, A. E.; Arris, C. E.; Bentley, J.; Boyle, F. T.; Curtin, N. J.; Davies, T. G.; Endicott, J. A.; Golding, B. T.; Grant, S.; Griffin, R. J.; Jewsbury, P.; Johnson, L. N.; Mesguiche, V.; Newell, D. R.; Noble, M. E.; Tucker, J. A.; and Whitfield, H. J. Probing the ATP ribose-binding domain of cyclin-dependent kinases 1 and 2 with $\mathrm{O}(6)$ substituted guanine derivatives. J. Med. Chem. 2002, 45, 3381-3393.

16. Davies, T. G.; Bentley, J.; Arris, C. E.; Boyle, F. T.; Curtin, N. J.; Endicott, J. A.; Gibson, A. E.; Golding, B. T.; Griffin, R. J.; Hardcastle, I. R.; Jewsbury, P.; Johnson, L. N.; Mesguiche, V.; Newell, D. R.; Noble, M. E.; Tucker, J. A.; Wang, L.; and Whitfield, H. J. Structure-based design of a potent purine-based cyclin-dependent kinase inhibitor. Nat. Struct. Biol. 2002, 9, 745-749.

17. Lowe, E. D.; Tews, I.; Cheng, K. Y.; Brown, N. R.; Gul, S.; Noble, M. E.; Gamblin, S. J.; and Johnson, L. N. Specificity determinants of recruitment peptides bound to phosphoCDK2/cyclin A. Biochemistry 2002, 41, 15625-15634. 
18. Schulze-Gahmen, U.; De Bondt, H. L.; and Kim, S. H. High-resolution crystal structures of human cyclin-dependent kinase 2 with and without ATP: bound waters and natural ligand as guides for inhibitor design. J. Med. Chem. 1996, 39, 4540-4546.

19. Schulze-Gahmen, U.; Brandsen, J.; Jones, H. D.; Morgan, D. O.; Meijer, L.; Vesely, J.; and Kim, S. H. Multiple modes of ligand recognition: crystal structures of cyclindependent protein kinase 2 in complex with ATP and two inhibitors, olomoucine and isopentenyladenine. Proteins 1995, 22, 378-391.

20. Russo, A. A.; Jeffrey, P. D.; and Pavletich, N. P. Structural basis of cyclin-dependent kinase activation by phosphorylation. Nat. Struct. Biol. 1996, 3, 696-700.

21. Clare, P. M.; Poorman, R. A.; Kelley, L. C.; Watenpaugh, K. D.; Bannow, C. A.; and Leach, K. L. The cyclin-dependent kinases cdk2 and cdk5 act by a random, anticooperative kinetic mechanism. J. Biol. Chem. 2001, 276, 48292-48299.

22. Furet, P.; Meyer, T.; Strauss, A.; Raccuglia, S.; and Rondeau, J. M. Structure-based design and protein X-ray analysis of a protein kinase inhibitor. Bioorg. Med. Chem. Lett. 2002, 12, 221-224.

23. Bramson, H. N.; Corona, J.; Davis, S. T.; Dickerson, S. H.; Edelstein, M.; Frye, S. V.; Gampe, R. T., Jr.; Harris, P. A.; Hassell, A.; Holmes, W. D.; Hunter, R. N.; Lackey, K. E.; Lovejoy, B.; Luzzio, M. J.; Montana, V.; Rocque, W. J.; Rusnak, D.; Shewchuk, L.; Veal, J. M.; Walker, D. H.; and Kuyper, L. F. Oxindole-based inhibitors of cyclindependent kinase 2 (CDK2): design, synthesis, enzymatic activities, and X-ray crystallographic analysis. J. Med. Chem. 2001, 44, 4339-4358.

24. Sayle, K. L.; Bentley, J.; Boyle, F. T.; Calvert, A. H.; Cheng, Y.; Curtin, N. J.; Endicott, J. A.; Golding, B. T.; Hardcastle, I. R.; Jewsbury, P.; Mesguiche, V.; Newell, D. R.; Noble, M. E.; Parsons, R. J.; Pratt, D. J.; Wang, L. Z.; and Griffin, R. J. Structure-based 


\section{Supporting Information}

design of 2-arylamino-4-cyclohexylmethyl-5-nitroso-6-aminopyrimidine inhibitors of cyclin-dependent kinases 1 and 2. Bioorg. Med. Chem. Lett. 2003, 13, 3079-3082.

25. Anderson, M.; Beattie, J. F.; Breault, G. A.; Breed, J.; Byth, K. F.; Culshaw, J. D.; Ellston, R. P.; Green, S.; Minshull, C. A.; Norman, R. A.; Pauptit, R. A.; Stanway, J.; Thomas, A. P.; and Jewsbury, P. J. Imidazo[1,2-a]pyridines: a potent and selective class of cyclin-dependent kinase inhibitors identified through structure-based hybridisation. Bioorg. Med. Chem. Lett. 2003, 13, 3021-3026.

26. De Moliner, E.; Brown, N. R.; and Johnson, L. N. Alternative binding modes of an inhibitor to two different kinases. Eur. J. Biochem. 2003, 270, 3174-3181.

27. Johnson, L. N., De Moliner, E., Brown, N. R., and Song, H. The Crystal Structure of Ucn-01 in Complex with Phospho-Cdk2/Cyclin A. Unpublished results.

28. Wu, S. Y.; McNae, I.; Kontopidis, G.; McClue, S. J.; McInnes, C.; Stewart, K. J.; Wang, S.; Zheleva, D. I.; Marriage, H.; Lane, D. P.; Taylor, P.; Fischer, P. M.; and Walkinshaw, M. D. Discovery of a novel family of CDK inhibitors with the program LIDAEUS: structural basis for ligand-induced disordering of the activation loop. Structure. (Camb. ) 2003, 11, 399-410.

29. Brown, N. R.; Noble, M. E.; Endicott, J. A.; and Johnson, L. N. The structural basis for specificity of substrate and recruitment peptides for cyclin-dependent kinases. Nat. Cell Biol. 1999, 1, 438-443.

30. Obermann, W. M.; Sondermann, H.; Russo, A. A.; Pavletich, N. P.; and Hartl, F. U. In vivo function of Hsp90 is dependent on ATP binding and ATP hydrolysis. J. Cell Biol. 1998, 143, 901-910. 
31. Jez, J. M.; Chen, J. C.; Rastelli, G.; Stroud, R. M.; and Santi, D. V. Crystal structure and molecular modeling of 17-DMAG in complex with human Hsp90. Chem. Biol. 2003, 10, 361-368.

32. Stebbins, C. E.; Russo, A. A.; Schneider, C.; Rosen, N.; Hartl, F. U.; and Pavletich, N. P. Crystal structure of an Hsp90-geldanamycin complex: targeting of a protein chaperone by an antitumor agent. Cell 1997, 89, 239-250.

33. Wright, L.; Barril, X.; Dymock, B.; Sheridan, L.; Surgenor, A.; Beswick, M.; Drysdale, M.; Collier, A.; Massey, A.; Davies, N.; Fink, A.; Fromont, C.; Aherne, W.; Boxall, K.; Sharp, S.; Workman, P.; and Hubbard, R. E. Structure-activity relationships in purinebased inhibitor binding to HSP90 isoforms. Chem. Biol. 2004, 11, 775-785. 